\title{
miR-1228 promotes the proliferation and metastasis of hepatoma cells through a p53 forward feedback loop
}

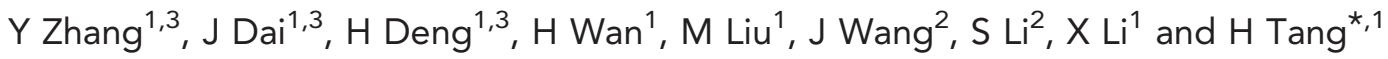 \\ ${ }^{1}$ Tianjin Life Science Research Center, Department of Microbiology, School of Basic Medical Sciences, Tianjin Medical University, \\ Tianjin 30070, China and ${ }^{2}$ State Key Laboratory of Oncology in Southern China, Department of Hepatobiliary Oncology, \\ Cancer Center, Sun Yat-sen University, Guangzhou 510060, China
}

Background: The effective mechanisms of microRNAs (miRNAs) functions as oncogenes or tumour suppressors in human hepatocellular carcinoma (HCC) are still obscure. Here, we investigated the function and expression of miR-1228 in HCC.

Methods: The role of miR-1228 in HCC was determined by colony formation, transwell, and nude mice xenograft experiments. miR-1228 target gene were identified by EGFP reporter assays, real-time PCR, and western blot analysis. Dual-luciferase reporter assay and real-time PCR analysis are used to examine the regulation of p53.

Results: miR-1228 promoted proliferation and metastasis, and facilitated the transition of cell cycle in hepatoma cells. miR-1228 downregulated p53 expression by binding to its $3^{\prime} U T R$. The ectopic expression of p53 abrogated the phenotypes induced by miR1228. An inverse correlation existed between miR-1228 and p53 expression in hepatoma tissues compared with the adjacent tissues and three hepatoma cell lines. Moreover, we found that p53 suppressed the expression and promoter activity of miR-1228.

Conclusions: miR-1228 functions as an oncogene by promoting cell cycle progression and cell mobility and negatively regulates the expression of p53. p53 downregulation in turn leads to an increase in miR-1228 expression, thereby forming a positive feedback loop that contributes to cancerogenesis in HCC.

Hepatocellular carcinoma (HCC) is one of the most common malignant tumours of the liver. It is the sixth most common malignancy worldwide and the third cause of cancer-related mortality (Parkin et al, 2005; Fares and Peron, 2013). However, the origin and development of HCC are complex and still obscure. It has been revealed that the altered expression or activity of specific genes, including microRNAs (miRNAs), is involved in the pathogenesis of HCC (Wang et al, 2010; Xie et al, 2012; Yan et al, 2013).

microRNAs (miRNAs) are noncoding endogenous RNA molecules, 20-25 nucleotides in length, that posttranscriptionally regulate gene expression by specifically targeting the $3^{\prime}$ untranslated regions ( $3^{\prime} \mathrm{UTR}$ ) of messenger RNAs (mRNAs) (Bartel, 2004,
2009), thus regulating cell differentiation, development, proliferation, and apoptosis (Bueno et al, 2008; Schickel et al, 2008; Urbich et al, 2008; Lynam-Lennon et al, 2009). Some studies have shown that a variety of miRNAs are involved in cancerogenesis and function as oncogenes or tumour suppressors (Suzuki et al, 2009; Cho, 2010; Imam et al, 2010; Wang et al, 2011; Xu et al, 2013). In a recent report, miR-27a was shown to reverse multiple drug resistance in hepatocellular carcinoma cells by inhibiting the FZD7/ $\beta$-catenin pathway (Chen et al, 2013). miR-133a participates in the MAPK pathway by inhibiting the phosphorylation of ERK and MEK (Wang et al, 2013). The TGF-beta-mediated miR-34a regulates CCL22 to affect the metastasis of hepatitis B viruspositive HCC (Yang et al, 2012).

\footnotetext{
${ }^{*}$ Correspondence: Dr H Tang; E-mail: htang2002@yahoo.com

${ }^{3}$ These authors contributed equally to this work
} 
Additionally, many studies have indicated the close interactions between miRNAs and p53, as well as its involvement in the p53 network at various levels. p53, which is encoded by the TP53 gene, regulates many genes that are involved in cell cycle progression, DNA repair, apoptosis, and angiogenesis (Harris and Levine, 2005). p53 was discovered as a tumour suppressor, and extensive studies have been undertaken to investigate its function and its signalling pathway (Braithwaite and Prives, 2006; Levine et al, 2006; Vousden and Prives, 2009). Within the p53 network, p53 is directly regulated by several miRNAs and, in turn, joins in the regulation of many miRNAs. On one hand, as a transcription factor, p53 directly or indirectly regulates the transcription and maturation of a group of miRNAs to initiate various cellular responses. It was found that p53 could activate the miR-34a family, thus inducing cell apoptosis, cell cycle arrest, and cell aging (He et al, 2007). The binding sites for p53 and TATA-binding protein overlap within the miR-17-92 promoter, resulting in the inhibition of miR-17-92 transcription (Yan et al, 2009). On the other hand, miRNAs can regulate the activity of $\mathrm{p} 53$ by directly targeting the $3^{\prime} \mathrm{UTR}$ of its mRNA or by repressing its regulators in the cells. miR-125b and miR-504 was shown to repress p53 expression by directly targeting the $3^{\prime} \mathrm{UTR}$ of $\mathrm{p} 53$ and function in human cell lines (Le et al, 2009; Hu et al, 2010). Some miRNAs that directly target p53 are, in turn, regulated by $\mathrm{p} 53$, thus forming a positive or negative feedback loop in the p53 network; these miRNAs include miR-125b and miR-22, which inhibit the expression of p53, and p53 has been shown to repress the expression of these miRNAs (Boominathan, 2010; Boldrup et al, 2012), indicating that p53 could increase its expression by suppressing its negative regulators.

Here, we found that miR-1228 promotes cellular proliferation by accelerating the $\mathrm{G} 1$ to $S$ phase and the $S$ to $G 2$ phase transitions, and enhances the migration and invasion of HCC cells in vivo and in vitro. Furthermore, miR-1228 directly targets the p53 $3^{\prime} \mathrm{UTR}$, resulting in the downregulation of p53 mRNA and protein expression. Because p53 also acts as a transcription factor, we determined whether miR-1228 was a potential target of p53. Interestingly, we found that miR-1228 is located in an intron of the low-density lipoprotein receptor-related protein-1 (LRP1) gene, and discovered that $\mathrm{p} 53$ suppressed the promoter activity of miR1228. Thus, we concluded that miR-1228 participates in a p53positive feedback loop in HCC cell lines. Moreover, as an important component of the p53 network, inhibiting the expression of miR-1228 resulted in the accumulation of p53 expression. This study adds another layer of complexity to the p53 network in HCC and provides insight into the cancerogenesis of HCC.

\section{MATERIALS AND METHODS}

Cell culture and transfection. The human nontumourigenic hepatocyte cell line LO2 and hepatocelluar carcinoma cell lines QGY-7703 and HepG2 were maintained in RPMI 1640 (LO2, QGY-7703) and MEM- $\alpha$ (HepG2) (GIBCO). LO2 and QGY-7703 cell lines media was supplemented with $10 \%$ foetal bovine serum (while HepG2 used 20\% foetal bovine serum), $100 \mathrm{IU} \mathrm{ml}^{-1}$ of penicillin and $100 \mu \mathrm{g} \mathrm{ml}^{-1}$ of streptomycin, and incubated at $37^{\circ} \mathrm{C}$ in a humidified chamber supplemented with 5\% CO2. Transfection was performed with Lipofectamine 2000 Reagent (Invitrogen, Carlsbad, CA, USA) following the manufacturer's protocol.

RNA extract, quantitative reverse transcript-PCR (qRT-PCR), and western blot analysis. RNAs were isolated using mirVana miRNA Isolation Kit (Ambion, Austin, TX, USA), following the manufacturer's instructions. Target genes and controls were analysed by qRT-PCR using SYBR Premix Ex TaqTM (Promega, Madison, WI, USA). Western blot was used to detect protein. Details can be found in the Supplementary Material.
Promoter sequence prediction. We chose the highly repetitive sequence using the FirstEF, Promoter 2.0 Prediction Server, Berkeley Drosophila Genome Project Neural Network Promoter Prediction, and Promoter Scan programs.

miRNA target prediction. The analysis of miR-1228 predicted targets was performed using the TargetScan, PicTar, and miRBase Targets(Griffiths-Jones et al, 2006).

Vector construction. We constructed the miR-1228 expression vector (pcDNA3/pri-miR-1228), enhanced green fluorescent protein (EGFP) reporter plasmid with the wild-type p53 mRNA $3^{\prime} \mathrm{UTR}$ (pcDNA3/EGFP-p53-3'UTR) and with the mutated miR1228 binding site (pcDNA3/EGFP-p53-3'UTR mut.), pGL3-Basic$\mathrm{luc}^{+}$with the $866 \mathrm{bp}$ and $1546 \mathrm{bp}$ miR-1228 promoter regions (LRP1/miR-1228-p866 or LRP1/miR-1228-p1546) and the mutant vector LRP1/miR-1228-mut. All the details of construction can be found in the Supplementary Material.

Luciferase reporter assay. The cells were seeded in a 48 -well plate for $48 \mathrm{~h}$ after transfection with the two miR-1228 promoter reporter constructs, negative control vector containing the Renilla luciferase pRL-TK, or the positive control pGL3-control/luciferase vector to monitor which construct had the highest activity. The cells were then transfected with the miR-1228 promoter reporter construct or the mutant construct, along with shR-p53 or pcDNA3-p53 and their controls. Details can be found in the Supplementary Material.

Enhanced green fluorescent protein reporter assay. QGY7703 cells were cotransfected in 48-well plates with the reporter vector pEGFP-P53-3'UTR or pEGFP-P53-3'UTR-mut and pcDNA3/primiR-1228, pcDNA3, ASO-miR-1228 or ASO-NC, respectively. The vector pDsRed2-N1 (Clontech, Mountain View, CA, USA) expressing RFP was used as the internal control. After transfection for $48 \mathrm{~h}$, cells were lysed using RIPA lysis buffer $(150 \mathrm{~mm} \mathrm{NaCl}$, $50 \mathrm{~mm}$ Tris-HCl, pH 7.2, 1\% Triton X-100, and 0.1\% SDS) $200 \mu \mathrm{l}$ per well. The intensities of EGFP and RFP fluorescence were detected with Fluorescence Spectrophotometer F-4500 (HITACHI, Tokyo, Japan).

Detection of cell colony formation. For cell colony-formation assay, QGY-7703 cells were transfected with pri-miR-1228 and ASO-miR-1228 and controls. Details can be found in the Supplementary Material.

Migration and invasion assays. QGY-7703 cells were transfected with pri-miR-1228, ASO-miR-1228, or the corresponding controls. A 24-well Boyden chamber with an $8 \mathrm{~nm}$ pore size polycarbonate membrane (Millicell, Millipore, Merck KGaA, Darmstadt, Germany) was used to analyse the migration and invasion of the tumour cells. For the invasion assay, the membrane was coated with Matrigel (Clontech). Details can be found in the Supplementary Material.

Cell cycle analysis by flow cytometry. Transfected QGY-7703 cells were plated in duplicate in 6-well plates and incubated for $24 \mathrm{~h}$ in complete culture medium. One group of cells was deprived of serum for $24 \mathrm{~h}$ prior to harvest, whereas another group of cells was returned to complete medium for another $24 \mathrm{~h}$ before harvest. Details can be found in the Supplementary Material.

Immunohistochemistry. Tissues were fixed in phosphate-buffered neutral formalin, embedded in paraffin, and cut into $5-\mu \mathrm{m}$ thick sections. Tissue sections were deparaffinised, rehydrated, and microwave-heated in sodium citrate buffer for antigen retrieval. The sections were then incubated with $0.3 \%$ hydrogen peroxide/ PBS for $30 \mathrm{~min}$. Sections were stained with eosin (H\&E) and an anti-p53 antibody (Saierbio, Tianjin, China); the p53 antibody was used at a 1:50 dilution and was incubated with the slides overnight at $4{ }^{\circ} \mathrm{C}$. Detection of the p53 antibody was performed using goat 
anti-rabbit-HRP for $1 \mathrm{~h}$ at room temperature and was visualised using DAB substrate.

Clinical HCC specimens. Twenty paired human HCC and adjacent nontumour liver tissues were collected from the Cancer Center of Sun Yat-sen University of Medical Science. The samples, including the human liver tumour and adjacent nontumour tissue, were obtained from patients undergoing hepatectomy for HCC in accordance with the ethical standards of the institutional committee.

Statistical analysis. The data are reported as the mean \pm s.d. of at least three times of independent experiments with the double-sided Student's $t$-test. A $P$ value of less than 0.05 was considered significant.

\section{RESULTS}

miR-1228 promotes the growth of HCC cells and accelerates the G1/S and S/G2 phase transitions. To determine the impact of miR-1228 on the growth of HCC cells, we constructed a miR-1228 plasmid and used qRT-PCR to confirm the expression of pri-miR1228 and ASO-miR-1228 (2'-O-methyl-modified antisense oligonucleotide of miR-1228). The levels of miR-1228 increased approximately 1.8-fold and 2-fold in QGY-7703 and HepG2 cells transfected with pri-miR-1228, respectively, compared with the control vector (Figure 1A). In contrast, the levels of miR-1228 in the ASO-miR-1228-treated cells decreased by 60\% (QGY-7703) and $70 \%$ (HepG2) compared with transfection with the scrambled oligomer (ASO-NC) (Figure 1A). Then, the QGY-7703 and HepG2 cells were transfected with pri-miR-1228 and ASO-miR-1228 to explore the effects of miR-1228 on cell growth using a colonyformation assay. The results showed that the colony-formation rates of the QGY-7703 cells transfected with pri-miR-1228 were increased by approximately $70 \%-100 \%$ over those of the control groups, whereas inhibiting miR-1228 expression decreased the colony-formation rates by approximately $50 \%$ compared with the control groups (Figure 1B). Similar results were observed in the HepG2 cells, as shown in Supplementary Figure S1A.

To investigate the mechanisms underlying the regulation of cell growth, we examined the alterations in cell cycle progression caused by miR-1228 in the HCC cells. Flow cytometry analysis showed the effects of miR-1228 on cell cycle progression (Figure 1C). The overexpression of miR-1228 in QGY-7703 cells increased the percentage of cells in the $\mathrm{G} 2 / \mathrm{M}$ phase from $15.57 \%$ to $19.04 \%$ and decreased the percentage of cells in the G1/G0 phase from $60.21 \%$ to $52.02 \%$ (Figure 1D). The proliferation index of the pri-miR-1228-treated cells was apparently higher than that of the negative control (Figure 1E). In contrast, inhibition of miR-1228 by ASO in QGY-7703 cells led to an increase in the percentage of cells in the G1/G0 phase from $58.53 \%$ to $65.18 \%$ and a decrease in the percentage of cells in the G2/M phase from $15.75 \%$ to $10.84 \%$ (Figure 1D). The proliferation index of the miR-1228 ASO-treated QGY-7703 cells was decreased compared with the ASO control (Figure 1E). These results indicate that miR-1228 contributes to cell growth by accelerating the G1 to S phase and the S to G2 phase transitions in human HCC cells.

miR-1228 promotes migration/invasion and metastasis in vitro and in vivo. To determine whether miR-1228 could affect cell migration/invasion and the metastasis of HCC, we performed transwell assays without Matrigel to analyse cell motility, and the cell invasion ability was examined using transwell assays with Matrigel. The migration and invasion capacities of QGY-7703 cells transfected with pri-miR-1228 were increased by approximately 1.1 - and 0.75-fold, respectively, whereas transfection with ASOmiR-1228 reduced these capacities by approximately $80 \%$ and $70 \%$, respectively, when compared with the controls (Figure 2A and B). Similar results were observed in the HepG2 cells, with an increase in the pri-miR-1228-transfected cells by approximately 0.55 - and 0.8 -fold and a decrease in the ASO-miR-1228-transfected cells by $25 \%$ and $50 \%$ compared with the control groups (Supplementary Figure S1B and C), indicating that miR-1228 promoted both the migration and invasion of HCC cells in vitro.

We next explored the role of miR-1228 in HCC metastasis in vivo. We obtained QGY-7703 pooled clones (QGY-7703/miR1228 cells) that stably expressed higher levels of miR-1228 through G418 screening. The qRT-PCR analysis showed that miR-1228 expression increased by approximately 4.5 -fold in the QGY-7703/ miR-1228 cells compared with the QGY7703/pcDNA3 cells (Figure 2F, left). Next, the QGY-7703/miR-1228 cells and control pooled clones were transplanted into the upper poles of the spleen in nude mice. After 4 weeks, the spleens and livers were harvested (Figure 2C). In the respective groups of six nude mice, local cancers developed in the spleens of all of the mice, as shown by H\&E staining (Figure 2E), which also indicated the success of the mice xenograft metastasis model. Metastasis nodules on the liver surface were detected in the six mice of the QGY 7703/miR-1228 cell group, but were only detected in one mouse in the QGY7703/ pcDNA3 cell group (Figure 2C). The average number of metastatic nodules in the liver was dramatically increased, around six-fold (Figure 2D), in the groups with ectopic expression of miR-1228 (Figure 2F, right) compared with the control groups. Immunohistochemistry staining showed that $\mathrm{p} 53$ expression was reduced in the metastasis nodules derived from the QGY-7703 cells overexpressing miR-1228 compared with the control group (Figure 2G).

miR-1228 targets $p 53$ and negatively regulates its expression. miRNAs can potentially target multiple genes to elicit various phenotypes. To determine the target genes that mediate the effects of miR-1228 in HCC, we used bioinformatics analyses to determine that the $\mathrm{p} 53$ transcript has a putative miR-1228 binding site in its $3^{\prime} \mathrm{UTR}$ (Figure 3A). p53 was previously reported to suppress HCC proliferation (Lim et al, 2010) and mobility (Jing et al, 2005), and negatively regulate cell cycle (Lee et al, 2013) progression. Thus, we chose $p 53$ as a candidate target gene of miR1228 and investigated the interactions between miR-1228 and p53 mRNA.

First, we verified the effectiveness of shR-p53 and pcDNA3-p53 (Supplementary Figure S2A and B) and examined the negative regulation of $\mathrm{p} 53$ on the proliferation (Supplementary Figure S2C and D), migration, and invasion (Supplementary Figure S3A-D) of QGY-7703 and HepG2 cells. To explore a direct interaction between miR-1228 and the $3^{\prime} \mathrm{UTR}$ of the $p 53$ transcript, we performed EGFP reporter assays and found that co-transfection of the wild-type $3^{\prime}$ UTR of $p 53$ with pri-miR-1228 reduced the EGFP intensity by approximately $35 \%$, whereas transfection with ASOmiR-1228 caused an increase by $40 \%$ compared with the controls (Figure 3B). However, neither pri-miR-1228 nor ASO-miR-1228 had a significant effect on the EGFP reporter with the $p 533^{\prime} \mathrm{UTR}$ that contained a mutant miR-1228 binding site (Figure $3 \mathrm{~B}$ ).

Western blot and qRT-PCR analyses were used to examine the effect of miR-1228 on endogenous p53 expression. Ectopic expression of miR-1228 decreased the amount of p53 mRNA by $40-60 \%$ (Figure 3C) and of p53 protein by $30-40 \%$ (Figure 3D) in both the QGY-7703 and HepG2 cells compared with the control group. Opposite effects were observed in the ASO-miR1228-transfected cells at both the protein and mRNA levels (Figure 3C and D).

To explore the correlation between miR-1228 and p53 in HCC tissue, we examined 20 paired clinical specimens using qRT-PCR analysis. Compared with the adjacent nontumour tissues, miR1228 was upregulated in seven tumour tissues, in which p53 was 

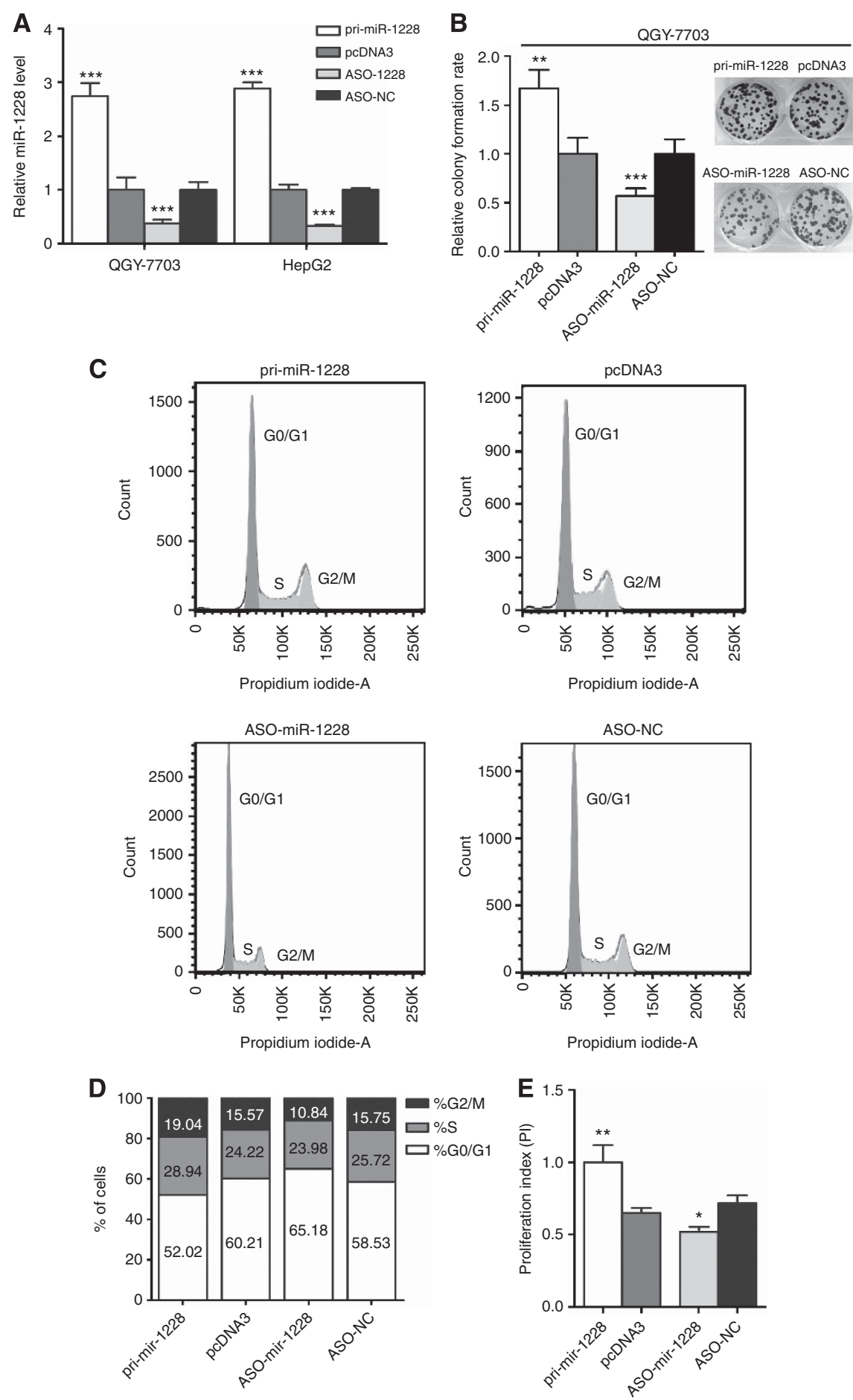

Figure 1. miR-1228 accelerates the $\mathrm{G} 1$ to $\mathrm{S}$ and the $\mathrm{S}$ to $\mathrm{G} 2$ phase transitions and promotes the proliferation of HCC cells. (A) QGY-7703 and HepG2 were transfected with pcDNA3/pri-miR-1228, ASO-miR-1228, or the negative controls, and miR-1228 was detected using qRT-PCR analysis; U6 snRNA was used for normalisation. (B) The colony-formation assay in the QGY-7703 cells revealed the relative average colonyformation rate. The colony-formation rate was calculated using the following equation: colony-formation rate $=$ (number of colonies/number of plated cells) $\times 100 \%$. (C) Distribution of cells in three phases (G0/G1, S, and G2/M) of the cell cycle as determined by flow cytometry analysis. (D) Cytometric quantification of the experiments described in the chart, showing the proportion of cells in the G0/G1, S, and G2/M phases. (E) The proliferation index $(\mathrm{PI})$ was calculated using the following equation: $\mathrm{PI}=(\mathrm{S}+\mathrm{G} 2) / \mathrm{G} 1$, where $\mathrm{S}, \mathrm{G} 2$, and $\mathrm{G} 1$ are the percentages of cells in the $\mathrm{S}$ phase, G2/M phase, and G0/G1 phase, respectively. ${ }^{\star} P<0.05 ;{ }^{\star \star} P<0.01$.

downregulated (Figure 3E). Then, we did the correlation analysis and found out miR-1228 and p53 had negative correlation in the 20 pairs primary HCCs (Figure 3E). We also detected the expression levels of miR-1228 and p53 in the QGY-7703 and HepG2 HCC cell lines and the immortalised normal hepatic LO2 cell line (Cheung et al, 2013). The miR-1228 expression level was upregulated in the QGY-7703 and HepG2 cells, whereas the p53 protein level was downregulated compared with the LO2 cells (Figure 3F). These results indicate that the expression of miR-1228 has an inverse relationship with p53 expression. 

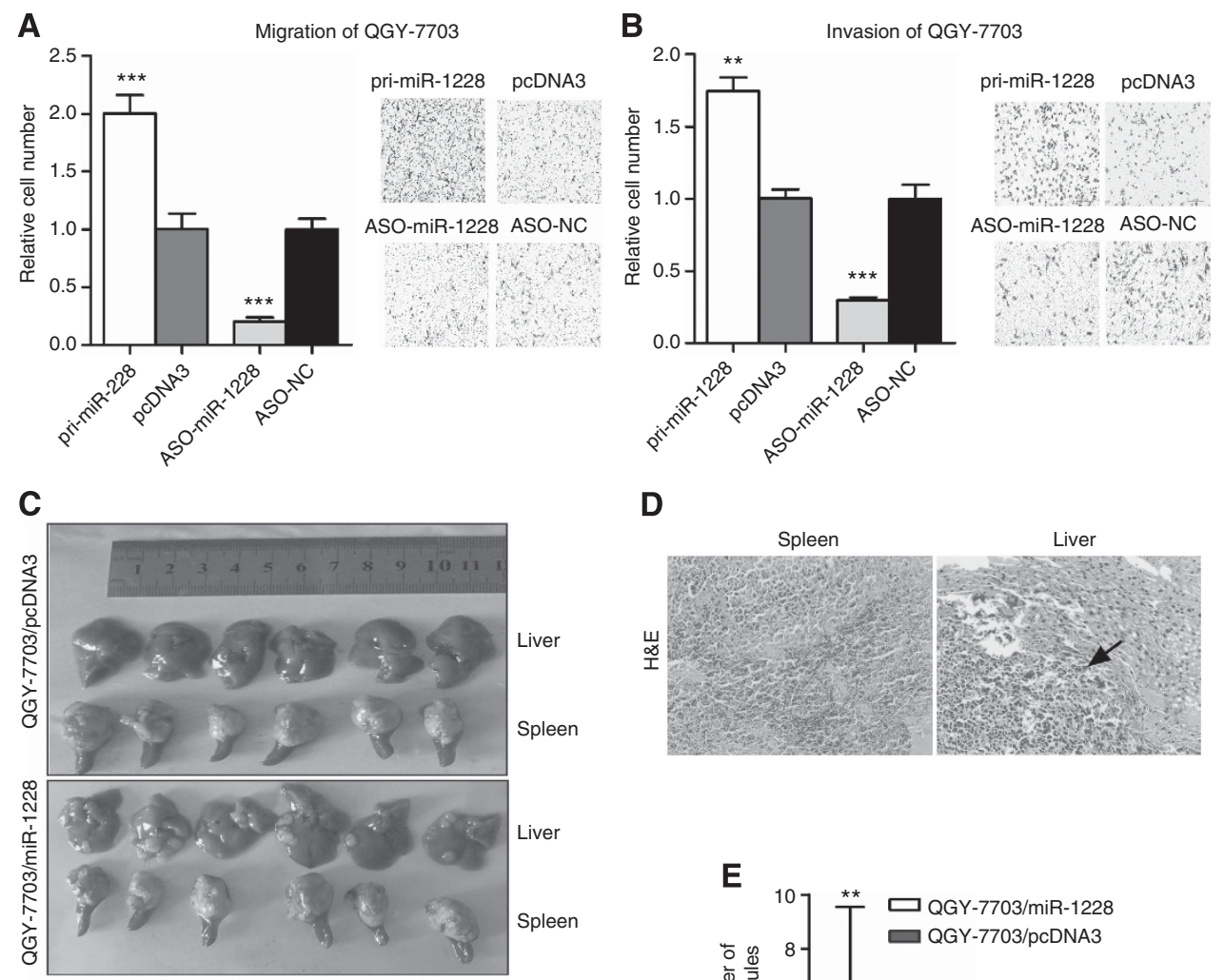

D
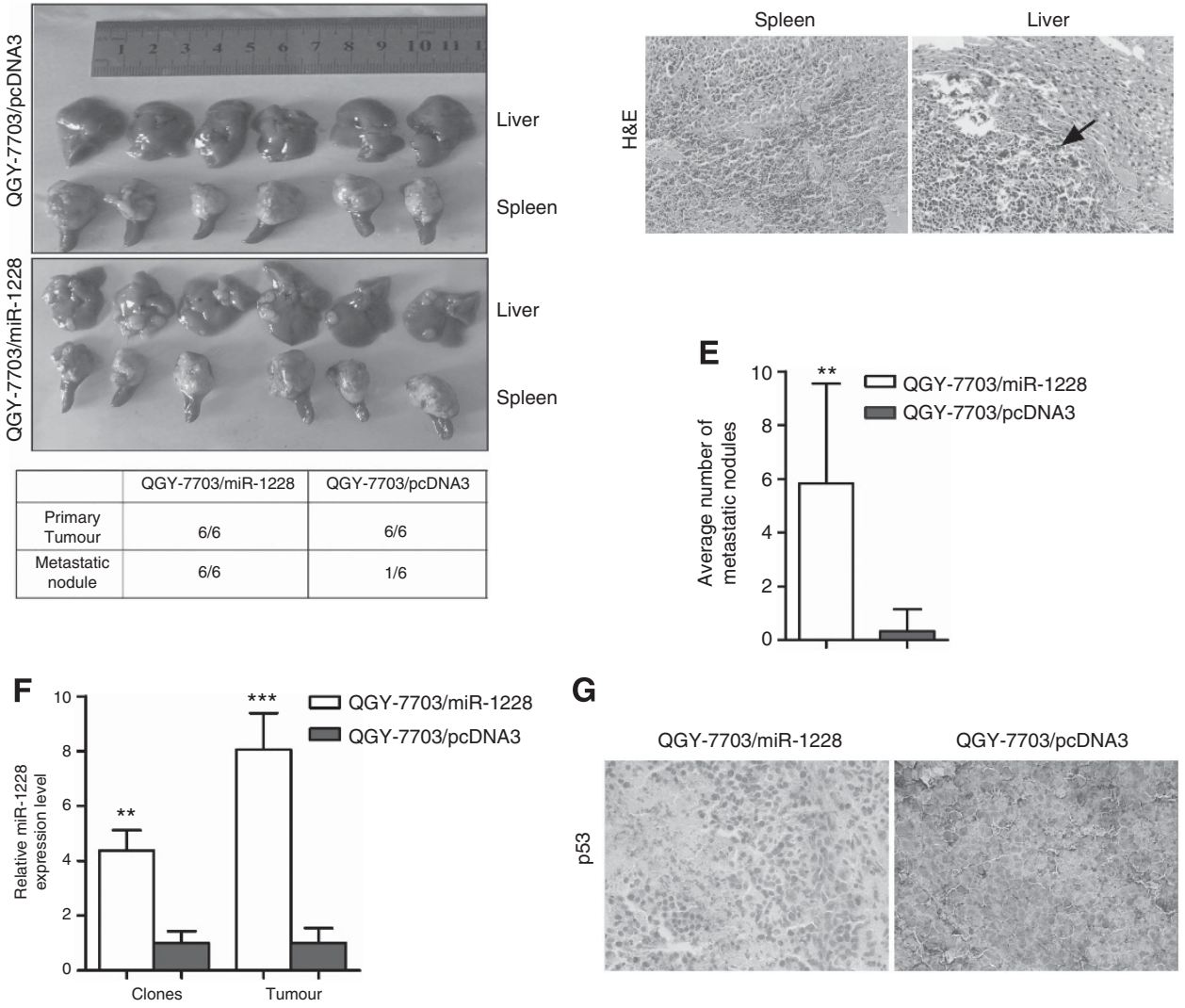

$\mathbf{G}$

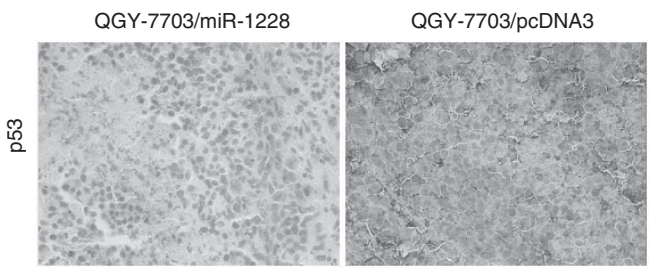

Figure 2. miR-1228 promotes the metastasis of HCC cells. (A and B) Transwell migration assays were carried out with QGY-7703 cells transfected with either pri-miR-1228 or ASO-miR-1228. Representative fields of the migratory and invasive cells on the membrane are presented. (C) A representative picture and table of the tumour nodules in the primary sites (spleen) and metastatic sites (liver) 4 weeks after spleen transplantation. (D) The scale bars indicate the clusters of metastatic cells. (E) Haematoxylin and eosin staining of the spleens (left) and livers (right) isolated from mice 4 weeks after the ectopic injection of QGY-7703 pooled clones expressing miR-1228 or pcDNA3. The black arrow indicates the HCC tissue of the liver. (F) Quantitative RT-PCR was used to detect the expression of miR-1228 in the QGY-7703 pooled clones and tumour nodules of the liver. (G) Immunohistochemical (IHC) staining of p53 in the sections of liver tumour formed from pooled clones of QGY-7703 cells expressing miR-1228 or pcDNA3 is also shown. ${ }^{\star} P<0.05$; ${ }^{\star \star} P<0.01$; ${ }^{\star \star \star} P<0.001$.

Restoration of p53 abrogates the effects induced by miR-1228 in HCC cells. Furthermore, to confirm that miR-1228's effect on $\mathrm{HCC}$ cells is due to its regulation of p53, we designed a series of experiments to determine whether the ectopic expression of $\mathrm{p} 53$ could counteract the proliferation and invasion of HCC cells caused by miR-1228. First, qRT-PCR and western blot analyses showed that p53 expression in HCC cells overexpressing miR-1228 was restored by co-transfection with pcDNA3-p53 compared with transfection with the control vector (Figure $4 \mathrm{~A}$ and $\mathrm{B}$ ). The miR1228-mediated promotion of colony formation in the QGY-7703 (Figure 4C) and HepG2 (Supplementary Figure S4A) cells was abolished by the ectopic expression of p53. Moreover, the migration and invasion capacities that were enhanced by miR1228 expression in the HCC cells were also counteracted by transfection with pcDNA3-p53 (Figure 4D and E). Similar results were observed in the HepG2 cells (Supplementary Figure S4B and C). These results demonstrate that p53 is the mediator of the miR1228-promoted cell proliferation and motility in HCC cells.

p53 inhibits the promoter activity of miR-1228 and negatively regulates miR-1228 expression in HCC cells. According to the bioinformatics analysis, we found that pri-miR-1228 is located in intron 49 of its host gene, LRP1 (Figure 5A), which has been shown to be important in liver metabolism (Basford et al, 2011). 
A
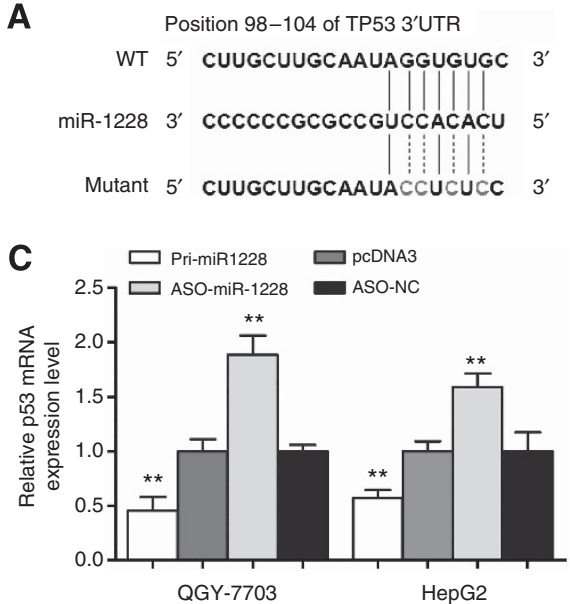

D $\square$ Pri-miR-1228 $\square$ pcDNA3

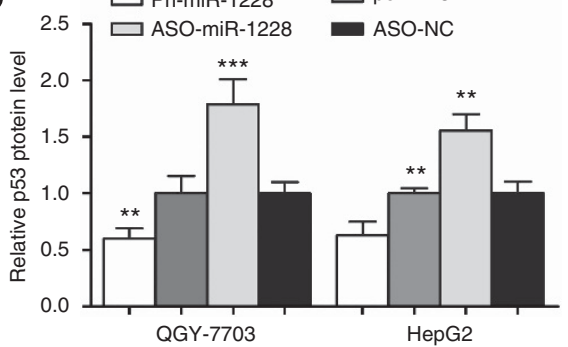

p53 (53 kDa) $\operatorname{GAPDH}(37 \mathrm{kDa}) \rightleftharpoons \infty$

$\mathbf{F}$

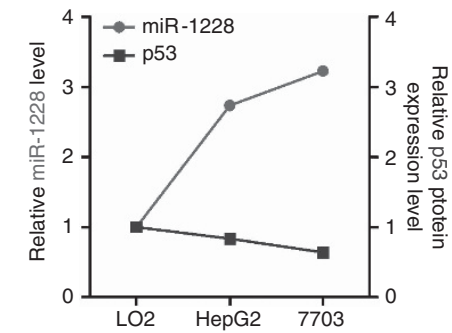

p53 (53 kDa)

GAPDH (37 kDa)
B

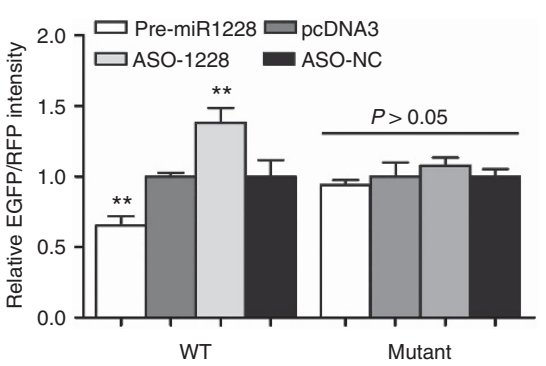

E
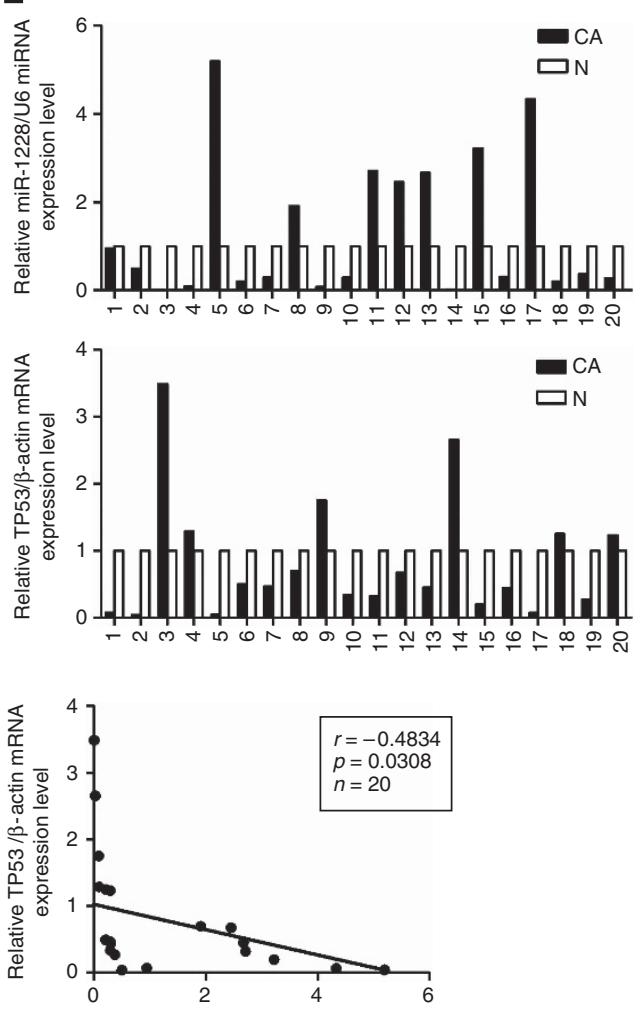

Relative miR-1228/U6 expression leve

Figure 3. miR-1228 negatively regulates the expression of p53 by directly targeting its $3^{\prime}$ UTR. (A) Sequence alignment of miR-1228 (middle) with the wild-type (WT) (top) and mutated (Mutant) (bottom) 3'UTR of the TP53 gene. The dotted lines indicate the mutated nucleotides. (B) The fluorescent reporter vectors (WT, Mutant) were co-transfected with pri-miR-1228 or ASO-miR-1228 into QGY-7703 and HepG2 cells, and the fluorescence intensity of the protein extract was determined. The EGFP fluorescence intensity was normalised to RFP. (C and D) The mRNA or protein levels of p53 were determined using qRT-PCR or western blot analysis in QGY-7703 and HepG2 cells, respectively. (E and F) The relative expression level of mature miR-1228 in 20 paired liver tissues, including HCC tissues (CA) and their adjacent noncancerous tissues (N), the HCC cell lines QGY-7703 and HepG2, and the immortalised normal hepatic cell line LO2, were detected using qRT-PCR analysis; U6 snRNA was used for normalisation. p53 mRNA and protein expression was detected using qRT-PCR and western blot analysis using $\beta$-actin and GAPDH for normalisation, respectively. In the correlation analysis, $r=-0.4834 .{ }^{\star} P<0.05 ;{ }^{\star \star} P<0.01$.

We predicted several putative p53-binding sites in the promoter region of LRP1. p53 is an important transcription factor that contributes to miRNA expression regulation (Tarasov et al, 2007). Thus, we speculated that p53 may regulate miR-1228 and its host gene LRP1. To examine this possibility, the gain and loss of p53 functions were used to study its influence on miR-1228 and LRP1. As shown in Figure $5 \mathrm{~B}$ and $\mathrm{C}$, the overexpression of $\mathrm{p} 53$ reduced the mRNA levels of both miR-1228 and LRP1 by approximately 40\%, whereas depletion of 553 resulted in increased mRNA levels of three-fold or five-fold in the QGY-7703 cells, respectively, which suggested that miR-1228 and its host gene LRP1 might be transcribed from the same promoter. To further confirm that p53 may regulate the expression of miR-1228 and LRP1, two fragments, one from
$-1328 \mathrm{bp}$ to $218 \mathrm{bp}$ and another one from $-1328 \mathrm{bp}$ to $-462 \mathrm{bp}$ upstream of the 5'UTR, were amplified from the putative promoter region using PCR and were cloned into the pGL3-Basic-luc ${ }^{+}$vector (LRP1/miR-1228-p1546 and LRP1/miR-1228-p866) (Figure 5A). Additionally, we made mutant constructs in which the putative p53-binding sites in LRP1/miR-1228-p866 or p1546 were altered (LRP1/miR-1228-mut) (Figure 5D). Luciferase activity in the transfected QGY-7703 cells was then detected using a dual-luciferase reporter assay. As shown in Figure 5E, the two promoter constructs behaved similarly: both LRP1/miR-1228-p866 and LRP1/miR-1228p1546 showed luciferase activities that were increased by 33 -fold and 23-fold, respectively, compared with the negative control vectors, which indicated that the two fragments possess promoter activity. 
A

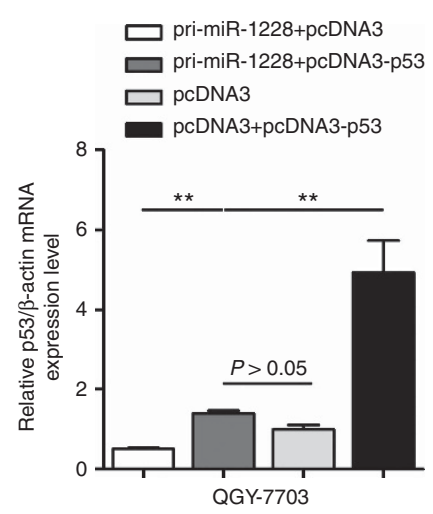

C

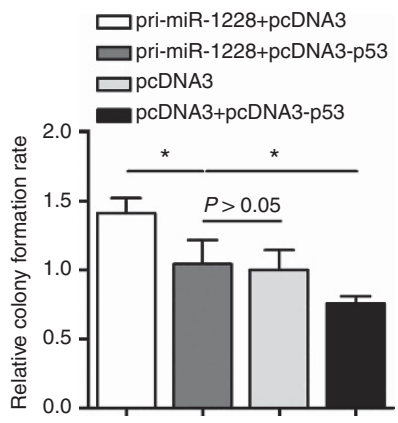

D

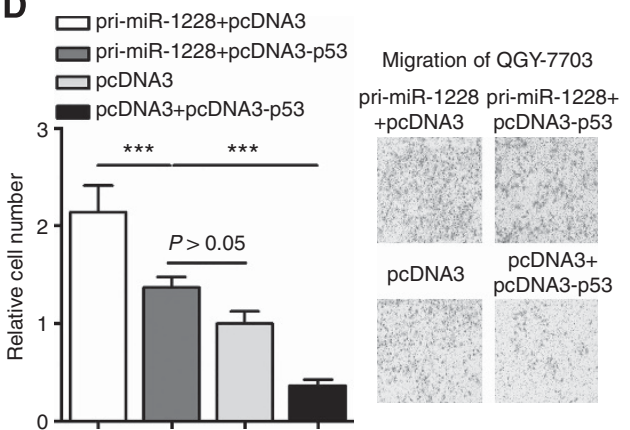

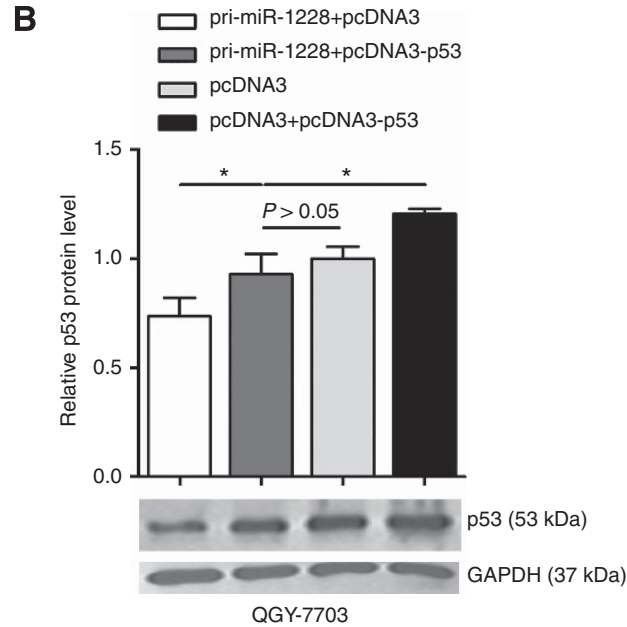
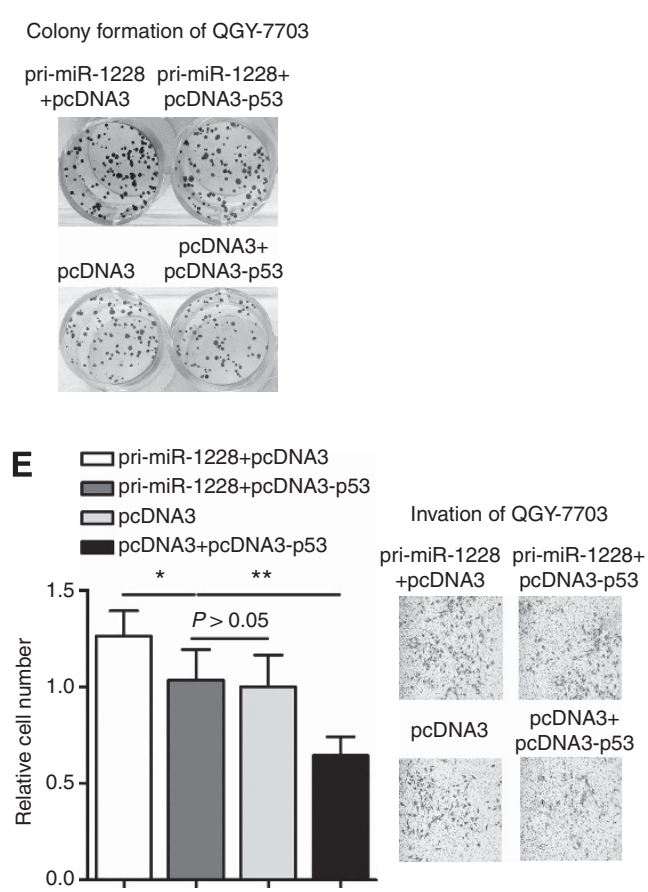

Figure 4. Restoration of p53 abrogates the effects induced by miR-1228 in QGY-7703 cells. (A and B) The mRNA and protein levels of p53 were tested using qRT-PCR or western blot analysis in QGY-7703 transfected with the vector shown in the figure. (C) The colony-formation assay revealed that the average colony-formation rate of QGY-7703 cells expressing miR-1228 was decreased following expression of p53 without a $3^{\prime}$ UTR. (D) Ectopic expression of p53 without a 3'UTR decreased the migration of the QGY-7703 cells when miR-1228 was present. (E) Similar assays for the cell invasion assay were found in the QGY-7703 cells. ${ }^{\star} P<0.05 ;{ }^{\star \star} P<0.01$.

Therefore, we performed the next experiments using the LRP1/miR1228 -p866 vector. Furthermore, we confirmed that p53 modulated the activity of the cloned promoter of LRP1/miR-1228. As shown in Figure 5F, the relative luciferase activity of p866 increased to 1.3-fold when co-transfected with shR-p53, but this activity decreased to approximately $65 \%$ in the pcDNA3-p53 group compared with the control groups. However, this effect was obviously impaired when cells were co-transfected with the reporter that contained a mutated p53-binding site (LRP1/miR-1228 mut) (Figure 5F). All together, these results demonstrate that p53 negatively regulates the transcription of miR-1228 and its host gene LRP1.

\section{DISCUSSION}

It is known that the participation of functional proteins and miRNAs varies in the p53 signalling pathway, making this pathway extremely complex. Previous studies have shown there are many functional downstream genes of p53. It has been reported that $\mathrm{p} 53$ can affect cell cycle-related proteins to regulate cell proliferation, such as activating the transcription of p21, p53R2, and GADD45 to suppress the progression of G1 (Vayssade et al, 2005). Additionally, by downregulating stathmin (Op18) or Lasp1 (Singer et al, 2007; Wang et al, 2009), p53 inhibits the metastasis of HCC cells. In HA22T/VGH and SK-Hep-1 cells, the low expression of PKC alpha leads to high p53 expression, which in turn inhibits the growth, migration, and invasion of these two cell lines (Wu et al, 2008). In BEC-7402 cells, VEGF165 promotes the growth and migration of HCC cells by negatively regulating the expression of p53 (Zhang et al, 2012). Therefore, p53 itself not only can be an effector of cell functions but also acts as a transcription factor to regulate its downstream genes. In the present study, we demonstrated that p53 suppresses miR-1228 expression, and the bioinformatics analysis showed that the miR-1228 gene is located 
A

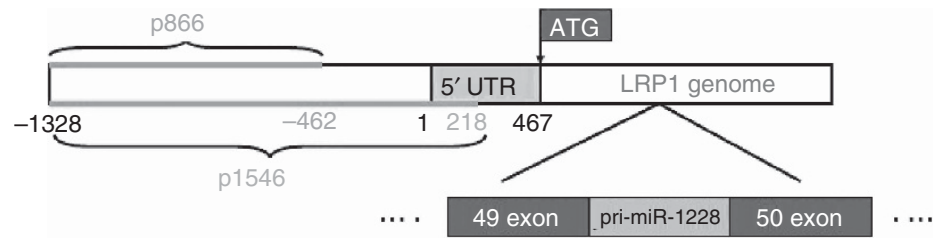

B

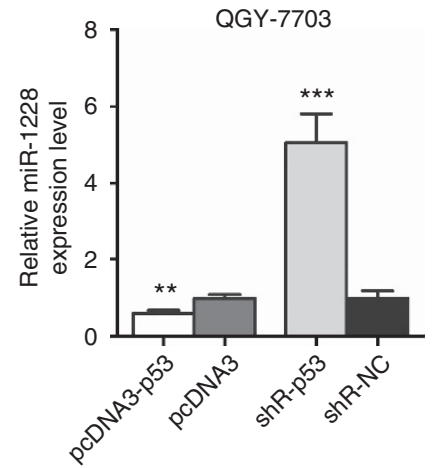

C

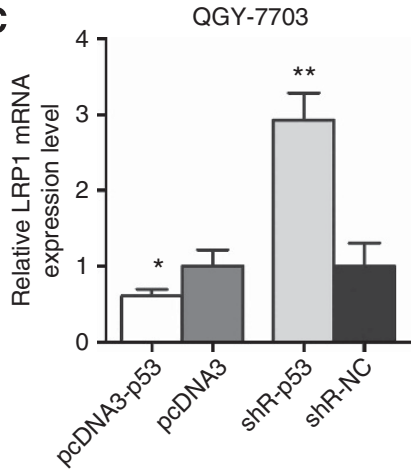

D

Position $-689 \mathrm{bp}$ to $-649 \mathrm{bp}$ of LRP1

5' GGCGGACAAGCTCCGGCGTGTCCCCTCGGGTGTCCCTGTT $3^{\prime}$

p53 binding site

mutant p53 binding site

5' GGCGTCGACAGACTCAGGTGTCCCCTCGGGACACTGTGTT $3^{\prime}$
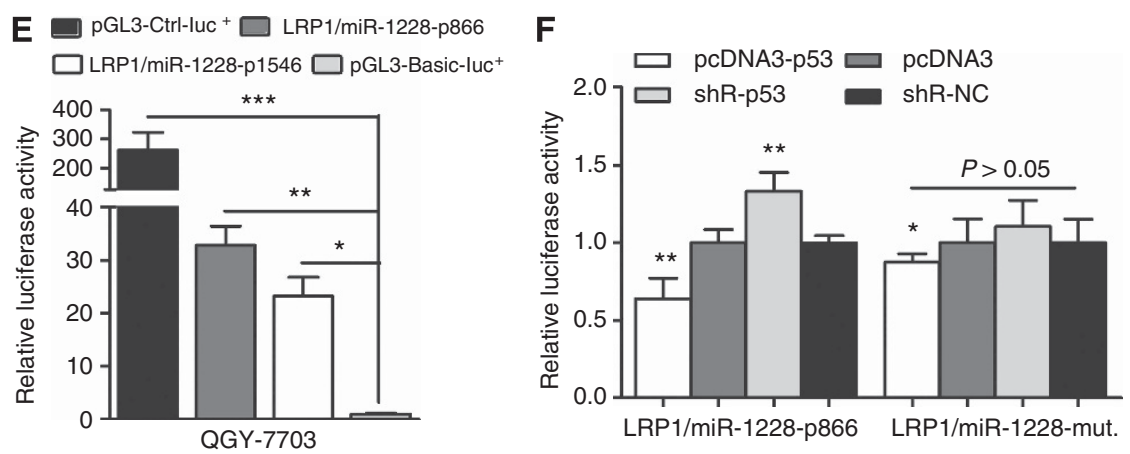

Figure 5. p53 suppresses miR-1228 promoter activity and negatively regulates its expression. (A) The diagram shows the location of pri-miR-1228 and the two fragments of the LRP1 promoter region. (B) miR-1228 was detected using qRT-PCR after overexpression or depletion of p53;

U6 snRNA was used for normalisation. (C) The LRP1 mRNA expression level was also detected using qRT-PCR, and $\beta$-actin was used to normalise mRNA levels after overexpression or knockdown of p53. (D) Two predicted binding sites were mutated, as shown in (E), the QGY-7703 cells were transiently transfected with the positive control pGL3-Ctrl-luc + , the negative control pGL3-Basic-luc + , or the LRP1/miR-p866 or p1645 constructs for $48 \mathrm{~h}$, followed by analysis with the dual-luciferase reporter assay. (F) The QGY-7703 cells were transiently transfected with LRP1/miR-1228-p866 or LRP1/miR-1228-mut. along with shR-p53 or pcDNA3-p53. ${ }^{\star} P<0.05 ;{ }^{\star \star} P<0.01 ;{ }^{\star \star \star} P<0.001$.

in an intron of the LRP1 gene. Thus, we speculated that p53 may exert its tumour suppressor role by modulating the expression of miR-1228 and its host gene LRP1. We cloned and analysed the promoter activity of the predicted promoter fragment for $L R P 1$ and miR-1228 from HCC cells. Utilizing the gain and loss of function by the ectopic overexpression and depletion of p53, as well as the presence of wild-type and mutant p53 predicted binding sites in the promoter fragment, p53 was shown to be a negative regulator in HCC cells. We also found that p53 expression is generally lower in HCC tissue compared with the adjacent noncancer tissues. It has been previously reported that p53 can repress the miR-17-92 cluster (Yan et al, 2009). Another study showed that although p53 cannot directly bind to the promoter region of RLIM, it can interact with $\mathrm{Sp} 1$ and prevent Sp1 binding to RLIM, thus inhibiting the Sp1-mediated transcriptional activation of RLIM (Kong et al, 2013). Thus, downregulation of p53 or p53 mutants may augment the expression of miR-1228 by modulating its promoter activity to contribute to cancerogenesis in HCC. To exert its tumoursuppressing effects, p53 controls the expression of miR-1228, which in turn impairs the expression of p53 and inhibits its tumour-suppressing function. Consequently, when we blocked the expression of miR-1228, p53 was released and enforced the inhibition of miR-1228, thereby promoting the accumulation of p53; however, miR-1228 is upregulated in HCC tissue in which p53 is downregulated, repressing p53 expression to relieve its inhibition on miR-1228 expression in turn, which creates a feedback regulation to ensure persistent low protein levels of p53 to control the malignant phenotype. Additionally, we further detected the mechanisms by which miR-1228 regulates the proliferation of HCC and found that miR-1228 could fasten cell cycle transitions.

Little is known about the functions of miR-1228 in cancer. A recent report showed that miR-1228 expression is high in 
malignant interstitial cell tumours compared with normal tissue (Guled et al, 2009), and this expression can inhibit stress-induced cellular apoptosis by directly interacting with the MOAP1 protein (Yan and Zhao 2012). More recently, miR-1228*, which is the complementary strand of the miR-1228 duplex, but not miR-1228, was found to be minimally expressed in human gastric cancer tissues, and this oligonucleotide inhibits the EMT and negatively regulates the activity of NF- $\kappa \mathrm{B}$, which make miR-1228* act as a tumour suppressor in gastric cancer (Jia et al, 2013). Here, we identified the functions of miR-1228 in HCC cells. The cell cycle analysis demonstrated that miR-1228 preferentially promotes cell proliferation by speeding the G1/S and S/G2 phage transitions of the cell cycle and promotes migration/invasion in vitro; metastasis experiments in an HCC xenograft model demonstrated that miR1228 facilitates the motility and metastasis of HCC cells in vivo. These roles of miR-1228 are contrary to the inherent function of p53 in HCC, and the bioinformatics analysis showed that the p53 $3^{\prime}$ UTR contains sites that are complementary to the miR-1228 seed sequence, suggesting that $\mathrm{p} 53$ is a potential miR-1228 direct target gene. Consequently, we confirmed that p53 is a direct target gene of miR-1228 using an EGFP reporter assay; qRT-PCR and western blot analyses demonstrated the inverse correlation between endogenous p53 and miR-1228 expression in HCC tissues and HCC cell lines. Thus, miR-1228 enhances cancer cell growth and metastasis through the partial downregulation of $\mathrm{p} 53$. However, we cannot exclude the possibility that other miR-1228 target genes mediate its effects in HCC.

In sum, our findings showed that miR-1228 may be a member of the p53 network and may interact with p53 at different levels in HCC. The downregulation of p53 results in an increase in the expression of miR-1228; in turn, miR-1228 targets and negatively regulates p53 expression, resulting in a forward feedback loop of $\mathrm{p} 53 / \mathrm{miR}-1228 / \mathrm{p} 53$. This regulatory network suggests that once this feedback loop forms, p53 expression will be maintained at a lower level to contribute to carcinogenesis, which may provide insight into the molecular mechanisms of the p53 signalling network during cancer initiation and development. Additionally, this feedback regulation suggests that miR-1228 may be a valuable biomarker for the development of diagnostic and treatment approaches for HCC.

\section{ACKNOWLEDGEMENTS}

This work was supported by the National Natural Science Foundation of China (No: 31270818; 30873017; 91029714; $31071191 ; 31101000)$ and the Natural Science Foundation of Tianjin (09JCZDJC17500; 12JCZDJC25100).

\section{CONFLICT OF INTEREST}

The authors declare no conflicts of interest.

\section{REFERENCES}

Bartel DP (2004) MicroRNAs: genomics, biogenesis, mechanism, and function. Cell 116(2): 281-297.

Bartel DP (2009) MicroRNAs: target recognition and regulatory functions. Cell 136(2): 215-233.

Basford JE, Wancata L, Hofmann SM, Silva RA, Davidson WS, Howles PN, Hui DY (2011) Hepatic deficiency of low density lipoprotein receptorrelated protein-1 reduces high density lipoprotein secretion and plasma levels in mice. J Biol Chem 286(15): 13079-13087.

Boldrup L, Coates PJ, Wahlgren M, Laurell G, Nylander K (2012) Subsite-based alterations in miR-21, miR-125b, and miR-203 in squamous cell carcinoma of the oral cavity and correlation to important target proteins. J Carcinog 11: 18.

Boominathan L (2010) The tumor suppressors p53, p63, and p73 are regulators of microRNA processing complex. PloS One 5(5): e10615.

Braithwaite AW, Prives CL (2006) p53: more research and more questions. Cell Death Differ 13(6): 877-880.

Bueno MJ, Perez de Castro I, Malumbres M (2008) Control of cell proliferation pathways by microRNAs. Cell Cycle 7(20): 3143-3148.

Chen Z, Ma T, Huang C, Zhang L, Lv X, Xu T, Hu T, Li J (2013) MiR-27a modulates the MDR1/P-glycoprotein expression by inhibiting FZD7/ beta-catenin pathway in hepatocellular carcinoma cells. Cell Signal 25(12): 2693-2701.

Cheung KF, Zhao J, Hao Y, Li X, Lowe AW, Cheng AS, Sung JJ, Yu J (2013) CITED2 is a novel direct effector of peroxisome proliferator-activated receptor gamma in suppressing hepatocellular carcinoma cell growth. Cancer 119(6): 1217-1226.

Cho WC (2010) MicroRNAs: potential biomarkers for cancer diagnosis, prognosis and targets for therapy. Int J Biochem Cell Biol 42(8): 1273-1281.

Fares N, Peron JM (2013) [Epidemiology, natural history, and risk factors of hepatocellular carcinoma]. La Revue du Praticien 63(2): 216-217, 220-212.

Griffiths-Jones S, Grocock RJ, van Dongen S, Bateman A, Enright AJ (2006) miRBase: microRNA sequences, targets and gene nomenclature. Nucleic Acids Res 34(Database issue): D140-D144.

Guled M, Lahti L, Lindholm PM, Salmenkivi K, Bagwan I, Nicholson AG, Knuutila S (2009) CDKN2A, NF2, and JUN are dysregulated among other genes by miRNAs in malignant mesothelioma -A miRNA microarray analysis. Genes Chromosomes Cancer 48(7): 615-623.

Harris SL, Levine AJ (2005) The p53 pathway: positive and negative feedback loops. Oncogene 24(17): 2899-2908.

He L, He X, Lim LP, de Stanchina E, Xuan Z, Liang Y, Xue W, Zender L, Magnus J, Ridzon D, Jackson AL, Linsley PS, Chen C, Lowe SW, Cleary MA, Hannon GJ (2007) A microRNA component of the p53 tumour suppressor network. Nature 447(7148): 1130-1134.

Hu W, Chan CS, Wu R, Zhang C, Sun Y, Song JS, Tang LH, Levine AJ, Feng Z (2010) Negative regulation of tumor suppressor p53 by microRNA miR504. Mol Cell 38(5): 689-699.

Imam JS, Buddavarapu K, Lee-Chang JS, Ganapathy S, Camosy C, Chen Y, Rao MK (2010) MicroRNA-185 suppresses tumor growth and progression by targeting the Sixl oncogene in human cancers. Oncogene 29(35): 4971-4979.

Jia L, Wu J, Zhang L, Chen J, Zhong D, Xu S, Xie C, Cai J (2013) Restoration of miR-1228* expression suppresses epithelial-mesenchymal transition in gastric cancer. PloS One 8(3): e58637.

Jing Z, Nan KJ, Hu ML (2005) Cell proliferation, apoptosis and the related regulators p27, p53 expression in hepatocellular carcinoma. World $J$ Gastroenterol 11(13): 1910-1916.

Kong X, Peng B, Yang Y, Zhang P, Qin B, Han D, Wang C, Dang Y, Liu JO, Yu L (2013) p53 Represses transcription of RING finger LIM domainbinding protein RLIM through Sp1. PLoS One 8(5): e62832.

Le MT, Teh C, Shyh-Chang N, Xie H, Zhou B, Korzh V, Lodish HF, Lim B (2009) MicroRNA-125b is a novel negative regulator of p53. Genes Dev 23(7): 862-876.

Lee SY, Lee GR, Woo DH, Park NH, Cha HJ, Moon YH, Han IS (2013) Depletion of Aurora A leads to upregulation of FoxO1 to induce cell cycle arrest in hepatocellular carcinoma cells. Cell Cycle 12(1): 67-75.

Levine AJ, Hu W, Feng Z (2006) The P53 pathway: what questions remain to be explored? Cell Death Differ 13(6): 1027-1036.

Lim SO, Kim H, Jung G (2010) p53 inhibits tumor cell invasion via the degradation of snail protein in hepatocellular carcinoma. FEBS Lett 584(11): 2231-2236.

Lynam-Lennon N, Maher SG, Reynolds JV (2009) The roles of microRNA in cancer and apoptosis. Biol Rev Camb Philos Soc 84(1): 55-71.

Parkin DM, Bray F, Ferlay J, Pisani P (2005) Global cancer statistics, 2002. CA: a cancer journal for clinicians 55(2): 74-108.

Schickel R, Boyerinas B, Park SM, Peter ME (2008) MicroRNAs: key players in the immune system, differentiation, tumorigenesis and cell death. Oncogene 27(45): 5959-5974.

Singer S, Ehemann V, Brauckhoff A, Keith M, Vreden S, Schirmacher P, Breuhahn K (2007) Protumorigenic overexpression of stathmin/Op18 by gain-of-function mutation in p53 in human hepatocarcinogenesis. Hepatology 46(3): 759-768. 
Suzuki HI, Yamagata K, Sugimoto K, Iwamoto T, Kato S, Miyazono K (2009) Modulation of microRNA processing by p53. Nature 460(7254): 529-533.

Tarasov V, Jung P, Verdoodt B, Lodygin D, Epanchintsev A, Menssen A, Meister G, Hermeking H (2007) Differential regulation of microRNAs by p53 revealed by massively parallel sequencing: miR-34a is a p53 target that induces apoptosis and G1-arrest. Cell Cycle 6(13): 1586-1593.

Urbich C, Kuehbacher A, Dimmeler S (2008) Role of microRNAs in vascular diseases, inflammation, and angiogenesis. Cardiovasc Res 79(4): 581-588.

Vayssade M, Haddada H, Faridoni-Laurens L, Tourpin S, Valent A, Benard J, Ahomadegbe JC (2005) P73 functionally replaces p53 in Adriamycintreated, p53-deficient breast cancer cells. Int J Cancer 116(6): 860-869.

Vousden KH, Prives C (2009) Blinded by the light: the growing complexity of p53. Cell 137(3): 413-431.

Wang B, Feng P, Xiao Z, Ren EC (2009) LIM and SH3 protein 1 (Lasp1) is a novel p53 transcriptional target involved in hepatocellular carcinoma. J Hepatol 50(3): 528-537.

Wang B, Hsu SH, Majumder S, Kutay H, Huang W, Jacob ST, Ghoshal K (2010) TGF beta-mediated upregulation of hepatic miR-181b promotes hepatocarcinogenesis by targeting TIMP3. Oncogene 29(12): 1787-1797.

Wang F, Li Y, Zhou J, Xu J, Peng C, Ye F, Shen Y, Lu W, Wan X, Xie X (2011) miR-375 is down-regulated in squamous cervical cancer and inhibits cell migration and invasion via targeting transcription factor SP1. Am J Pathol 179(5): $2580-2588$.

Wang H, An H, Wang B, Liao Q, Li W, Jin X, Cui S, Zhang Y, Ding Y, Zhao L (2013) miR-133a represses tumour growth and metastasis in colorectal cancer by targeting LIM and SH3 protein 1 and inhibiting the MAPK pathway. Eur J Cancer 49(18): 3924-3935.

Wu TT, Hsieh YH, Hsieh YS, Liu JY (2008) Reduction of PKC alpha decreases cell proliferation, migration, and invasion of human malignant hepatocellular carcinoma. J Cell Biochem 103(1): 9-20.

Xie Q, Chen XM, Lu FM, Zhang T, Hao ML, Wang YF, Zhao J, McCrae MA, Zhuang H (2012) Aberrant expression of microRNA 155 may accelerate cell proliferation by targeting sex-determining region $\mathrm{Y}$ box 6 in hepatocellular carcinoma. Cancer 118(9): 2431-2442.

Xu J, Li Y, Wang F, Wang X, Cheng B, Ye F, Xie X, Zhou C, Lu W (2013) Suppressed miR-424 expression via upregulation of target gene Chk1 contributes to the progression of cervical cancer. Oncogene 32(8): 976-987.

Yan B, Zhao JL (2012) miR-1228 prevents cellular apoptosis through targeting of MOAP1 protein. Apoptosis 17(7): 717-724.

Yan HL, Xue G, Mei Q, Wang YZ, Ding FX, Liu MF, Lu MH, Tang Y, Yu HY, Sun SH (2009) Repression of the miR-17-92 cluster by p53 has an important function in hypoxia-induced apoptosis. EMBO J 28(18): 2719-2732.

Yan Y, Luo YC, Wan HY, Wang J, Zhang PP, Liu M, Li X, Li S, Tang H (2013) MicroRNA-10a Is Involved in the Metastatic Process by Regulating Eph Tyrosine Kinase Receptor A4-Mediated Epithelial-Mesenchymal Transition and Adhesion in Hepatoma Cells. Hepatology 57(2): 667-677. Yang P, Li QJ, Feng Y, Zhang Y, Markowitz GJ, Ning S, Deng Y, Zhao J, Jiang S, Yuan Y, Wang HY, Cheng SQ, Xie D, Wang XF (2012) TGF-betamiR-34a-CCL22 signaling-induced Treg cell recruitment promotes venous metastases of HBV-positive hepatocellular carcinoma. Cancer Cell 22(3): 291-303.

Zhang L, Wang JN, Tang JM, Kong X, Yang JY, Zheng F, Guo LY, Huang YZ, Zhang L, Tian L, Cao SF, Tuo CH, Guo HL, Chen SY (2012) VEGF is essential for the growth and migration of human hepatocellular carcinoma cells. Mol Biol Rep 39(5): 5085-5093.

This work is published under the standard license to publish agreement. After 12 months the work will become freely available and the license terms will switch to a Creative Commons AttributionNonCommercial-Share Alike 3.0 Unported License.

Supplementary Information accompanies this paper on British Journal of Cancer website (http://www.nature.com/bjc) 